\title{
Démystifier la valeur cocréée digitale du consommateur : cadre et propositions fondées sur la théorie de la présence sociale
}

Article

Accepted Version

Hollebeek, L. D., Clark, M. K. and Macky, K. (2021)

Démystifier la valeur cocréée digitale du consommateur :

cadre et propositions fondées sur la théorie de la présence sociale. Recherche et Applications en Marketing (English Edition), 36 (4). pp. 27-47. ISSN 2051-5707 doi:

https://doi.org/10.1177/07673701211054200 Available at https://centaur.reading.ac.uk/101836/

It is advisable to refer to the publisher's version if you intend to cite from the work. See Guidance on citing.

To link to this article DOI: http://dx.doi.org/10.1177/07673701211054200

Publisher: Sage

All outputs in CentAUR are protected by Intellectual Property Rights law, including copyright law. Copyright and IPR is retained by the creators or other copyright holders. Terms and conditions for use of this material are defined in the End User Agreement. 


\section{www.reading.ac.uk/centaur}

\section{CentAUR}

Central Archive at the University of Reading

Reading's research outputs online 


\section{Démystifier la Valeur Cocréée Digitale du Consommateur:}

\section{Cadre et Propositions Fondés sur la Théorie de la Présence Sociale}

Alors que la recherche sur la cocréation prolifère, les études existantes ne parviennent pas à isoler sa manifestation à travers des plateformes digitales (par opposition à non-digitales). De plus, les recherches existantes explorent principalement le processus de cocréation (par rapport à son résultat de valeur cocréée), qui mérite donc un examen plus approfondi, en particulier dans le contexte digital. Sur la base de ces lacunes, nous explorons la valeur cocréée digitale du consommateur (CDCV), qui reflète la valeur perçue par le consommateur qui résulte de l'interaction, de la collaboration ou de la communication avec ou via des plateformes digitales. Nous classons les plateformes digitales en (i) plateformes homme-homme (H2HP; par exemple, les médias sociaux), et (ii) plateformes homme-machine (H2MP), qui comprennent les sous-types de (a) plateformes basées sur l'automatisation des processus robotiques (par exemple, centres d'appels), et (b) plateformes basées sur l'apprentissage automatique/en profondeur (par exemple, robots de service). Nous composons ensuite un cadre fondé sur la théorie de la présence sociale qui explore l'effet de l'intimité et de l'immédiateté perçues de la plateforme sur CDCV pour nos plateformes proposées. Nous formalisons les associations du cadre en développant des Propositions, et conclurons en discutant des implications importantes qui découlent de cette recherche.

Mots clés : Valeur cocréée digitale du consommateur (CDCV), plateforme digitale, théorie de la présence sociale, intelligence artificielle. 


\section{Introduction}

$\mathrm{Au}$ cours des deux dernières décennies, la cocréation (de valeur) a suscité un intérêt croissant des chercheurs et des praticiens (Ranjan et Read, 2016). En effet, «la [co]création de valeur... peut être considérée comme la raison d'être des relations de collaboration...» (Anderson, 1995, p. 349). En raison de sa capacité de création de valeur, les organisations de divers secteurs, notamment l'hôtellerie, l'éducation, la restauration rapide et les services professionnels, adoptent de plus en plus la cocréation comme indicateur de performance clé (Merz et al., 2018; Grissemann et Stokburger-Sauer, 2012). Par exemple, la plateforme digitale MyStarbucks est conçue pour traiter les commandes, personnaliser les activités des clients et offrir un accompagnement, cocréant ainsi de la valeur avec les clients (Fournier, 2019).

En parallèle, la recherche académique sur la cocréation prolifère, surtout du point de vue de S-D logic (Vargo et Lusch, 2016 ; Leclercq et al., 2016). Compte tenu de son noyau interactif, la cocréation peut émerger à travers différentes plateformes (par exemple, face à face/digitales; Keeling et al., 2019 ; Banker et al., 2011). Les plateformes sont des «points de contact physiques ou virtuels conçus pour fournir un soutien structurel à... l'échange» (Hollebeek, 2019, p. 89), qui facilitent la cocréation de valeur (Ramaswamy et Ozcan, 2016). Cependant, malgré les progrès significatifs réalisés dans la recherche sur la cocréation, la compréhension de la cocréation manifestée par les plateformes digitales est à la traîne, ce qui génère un important déficit de recherche (premier déficit de recherche identifié) sur ce sujet (Novani et Kijima, 2012 ; Füller, 2010). Les plateformes digitales sont des points de contact technologiques qui sont propices à la cocréation (Kannan et Li, 2017; Vanhouette, 2016; Breidbach et al., 2014), y compris les médias sociaux (par exemple, Facebook), l'économie de partage (par exemple, Uber), et des plateformes basées sur l'intelligence artificielle (par exemple, le robot Nao), pour n'en nommer que quelques-unes. En particulier, les recherches existantes sur la cocréation digitale ont eu tendance à aborder un contexte digital unique, cloisonné ou isolé (par exemple, les médias sociaux; He et Wan, 2015). Ainsi, malgré l'importance toujours croissante de la technologie (Drucker, 2011), les études, comme le propose cet article, qui explorent la dynamique de la cocréation sur différentes plateformes digitales restent rares.

De plus, bien que la recherche sur la cocréation soit florissante (Balaji et Roy, 2017), les études antérieures accordent une attention limitée au résultat de la cocréation basé sur la valeur de la valeur cocréée, révélant un deuxième déficit de recherche. C'est pour cette raison que, alors que la cocréation se concentre sur le processus interactif de création de valeur des acteurs (Dampérat et al., 2019), la valeur cocréée émerge comme le résultat perçu basé sur la valeur (c'est-à-dire, la valeur générée de manière interactive) de ce processus (Busser et Shulga, 2018; Black et Gallan, 2015). 
Compte tenu de la rareté relative de la recherche sur la valeur cocréée à ce jour, nous abordons ce concept en nous concentrant sur son sous-ensemble technologique de valeur cocréée digitale perçue par le consommateur (CDCV; Gyrd-Jones et Kornum, 2013), conformément au premier déficit de recherche que nous avons identifié ci-dessus. Bien que CDCV reflète la valeur perçue créée par l'interaction avec les points de contact technologiques, le rôle de la plateforme digitale dans la promotion de la valeur cocréée reste toujours mal identifié, ce qui justifie une exploration plus approfondie. Semblable à l'enquête de Kannan et Li (2017) sur le marketing digital en tant que sousensemble du marketing, cet article conceptuel examine donc la CDCV comme un sous-ensemble de valeur cocréée (c'est-à-dire, la valeur cocréée via des plateformes digitales). Autrement dit, contrairement à une valeur cocréée plus large qui peut être générée via des plateformes (non)digitales, la valeur cocréée digitale nécessite l'émergence d'une ou de plusieurs plateformes digitales.

Compte tenu de notre vision des plateformes digitales comme des points de contact technologiques (Breidbach et al., 2014), nous ne nous concentrons pas sur la notion connexe d'entreprise de type plateforme digitale, qui capture le modèle commercial des entreprises (en ligne) qui interviennent dans l'échange consommateur/fournisseur (par exemple, AirBnB; Täuscher et Laudien, 2017). Alors que les entreprises de type plateforme digitale déploient des plateformes digitales spécifiques (par exemple, l'application Uber; Smedlund, 2012), nous nous concentrons uniquement sur les interactions des consommateurs avec ces points de contact et leurs effets respectifs sur la valeur cocréée digitale (Tu et Zhang, 2013; Breidbach et al. , 2014).

Étant donné que les plateformes digitales existent avec une grande diversité, nous développons une typologie de plateformes digitales et évaluons la capacité de chaque plateforme à générer une valeur cocréée digitale. Bien que la littérature existante propose plusieurs classifications connexes, aucune de celles-ci ne se concentre spécifiquement sur les caractéristiques technologiques d'une plateforme donnée et leurs implications pour la valeur cocréée digitale, comme cela est entrepris ici. Par exemple, alors que la «typologie à quatre parties » (four-partite typology) de Wünderlich et al. (2013) classe les services interactifs intelligents en fonction d'une activité faible/élevée des utilisateurs et des fournisseurs, leur concentration sur l'engagement humain (c'est-à-dire, utilisateur/fournisseur), comme Füller (2010), empêche une évaluation directe du rôle des caractéristiques spécifiques de la plateforme digitale dans la création de valeur cocréée de ces services. De plus, Bolton et Saxena-Iyer (2009) catégorisent la mesure dans laquelle les services sont activés/fournis par la technologie (faible/élevé) sous des niveaux bas/élevés de participation des clients, respectivement, ce qui donne une typologie d'interactivité de service en quatre parties. Si la première dimension concerne en effet la technologie liée aux plateformes, elle évalue l'étendue de la 
technologie déployée, plutôt que ses caractéristiques particulières, ce que propose justement notre classification. En nous inspirant de la théorie de la présence sociale, nous développons également un cadre qui explore les effets respectifs de nos plateformes sur la valeur digitale cocréée par le consommateur, suivi par le développement d'une série de Propositions qui résument les associations proposées par notre model.

Cet article conceptuel propose deux contributions principales. Tout d'abord, après un examen approfondi, nous développons une typologie des plateformes digitales. Les typologies théoriques sont des classifications utilisées pour comprendre les catégories caractérisant des phénomènes d'intérêt spécifiques (Hambrick, 1984). Notre typologie de plateformes digitales comprend: (i) des plateformes homme-homme (par exemple, des applications mobiles) et (ii) des plateformes homme-machine, qui contiennent les sous-catégories de (a) plateformes basées sur l'automatisation des processus robotiques (par exemple, centres d'appels) et (b) des plateformes basées sur l'apprentissage automatique/en profondeur (deep learning; par exemple, les robots de service; Pradeep et al., 2019). Nous envisageons une dynamique CDCV unique pour ces catégories typologiques, comme discuté plus loin ci-dessous, offrant ainsi un tremplin important pour de futures recherches empiriques.

Deuxièmement, nous développons un cadre de CDCV fondé sur la théorie de la présence sociale, répondant aux appels de la littérature pour un cadre de valeurs cocréé rigoureux (Black et Gallan, 2015; Huber et al., 2017). En examinant la capacité des plateformes interactives à transmettre des indices sociaux, la théorie de la présence sociale propose l'existence de différences entre plateformes en termes de capacité à favoriser l'intimité et l'immédiateté perçues par l'utilisateur (Short et al., 1976), affectant ainsi de manière différentielle la CDCV. Alors que l'intimité et l'immédiateté sont optimisées dans les interactions en face à face, nous nous concentrons sur leur dynamique lors de l'utilisation de différentes plateformes digitales (Van Doorn et al., 2017). Nous adoptons une perspective de théorie de la présence sociale, étant donné qu'elle se concentre sur l'identification des différences trans-plateformes et leurs effets respectifs sur la CDCV, apportant ainsi une contribution importante. Bien que la S-D logic représente la principale perspective théorique de la cocréation (par exemple, Vargo et Lusch, 2016), sa nature purement abstraite et métathéorique l'empêche de mettre en évidence les caractéristiques des plateformes trans-digitales et leurs implications pour la CDCV. Nous modélisons donc la CDCV dans un cadre fondé sur la théorie de la présence sociale, suivant la position de MacInnis (2011, p. 141) selon laquelle la connaissance progresse «en conceptualisant la relation [d'un concept] à d'autres concepts, souvent dans un réseau nomologique». Dans le même ordre d'idée, nous développons des Propositions qui formalisent les associations du model proposé 
pour les plateformes digitales identifiées. Collectivement, nos analyses offrent un catalyseur pour de futures recherches empiriques sur la CDCV et un aperçu managérial de la CDCV.

Les sections suivantes se déroulent comme suit. Nous commençons par passer en revue la littérature sur la cocréation/valeur cocréée, qui offre une base importante pour nos analyses. Nous développons ensuite une typologie de plateforme digitale, suivie de la composition d'un model de CDCV fondé sur la théorie de la présence sociale. Sur la base de ce model, nous élaborons des Propositions, suivi d'un aperçu des implications, des limites et des pistes de recherche de cette étude.

\section{Revue de Littérature}

\section{Cocréation}

La plupart des recherches publiées portent sur le processus de cocréation, plutôt que sur le résultat basé sur la valeur de ce processus (Black et Gallan, 2015), comme indiqué précédemment. Ainsi, alors que la cocréation se déroule comme une séquence d'étapes (Buonincontri et al., 2017; Alves et al., 2018; Vega-Vazquez et al., 2013), la valeur cocréée reflète le niveau de valeur perçue par l'acteur (par exemple, le consommateur) qui découle de ces événements (Huber et al., 2017; Mahr et al., 2014). Étant donné la rareté relative de la recherche sur la valeur cocréée, nous passons en revue la littérature sur la cocréation, suivie d'une revue des travaux publiés sur la valeur cocréée. Sur la base de ces analyses, nous proposons une conceptualisation de la CDCV à la fin de la section suivante.

Avec ses fondements (par exemple, la coproduction) émergeant au milieu/à la fin des années 1990 (Normann et Ramírez, 1994; McColl-Kennedy et al., 2012), le concept de cocréation a été inventé pour la première fois au début des années 2000 (Nicod et Llosa, 2018; Dong et Sivakumar, 2017). Par exemple, l'expérience de cocréation de Prahalad et Ramaswamy (2004) offre l'expérience client de l'offre (par rapport à l'offre elle-même) en tant que fondement de la valeur perçue, semblable à la valeur d'utilisation (par rapport à la valeur d'échange; $\mathrm{Ng}$ et al., 2011; Humphreys et Grayson, 2008). Ici, la valeur perçue implique le compromis coût/bénéfice interne du consommateur lié à l'offre (Zeithaml, 1988; Woodruff, 1997).

En parallèle, Vargo et Lusch (2004) ont intégré la cocréation dans leur perspective S-D logic émergente. Ces auteurs considèrent les acteurs comme des contributeurs actifs (vs passifs) à leur propre création de valeur (Mahr et al., 2014; Schau et al., 2009). Lorsqu'une telle création de valeur se produit avec, à travers d'autres, ou pour d'autres (par exemple, via des interactions communautaires en ligne), elle est interprétée comme une cocréation. Prahalad et Ramaswamy (2000, p. 80) sont d'accord: «De plus en plus, [les consommateurs] veulent façonner [c'est-à-dire, cocréer... la valeur] avec des experts ou d'autres clients.» 
Bien que la recherche pionnière sur la cocréation n'ait pas été liée à la S-D logic en soi, au fil du temps, le concept est devenu de plus en plus lié à ce concept (Dahl et al., 2019; CW, 2020). Par exemple, la cocréation ne figure pas dans les Prémisses originales de S-D logic (Vargo et Lusch, 2004). Cependant, quatre ans plus tard, les auteurs l'intègrent dans FP6, qui déclare: «Le client est toujours un cocréateur de valeur» (Vargo et Lusch, 2008, p. 7). Pourtant, la cocréation n'a toujours pas de définition formelle dans ces articles (CW, 2020). En 2016, Vargo et Lusch définissent toutefois la cocréation comme «les actions d'acteurs multiples, souvent inconscients les uns des autres, qui contribuent au bien-être de l'autre» (p. 8). Ils révisent également FP6 (p. 8) comme suit: «La valeur est cocréée par de multiples acteurs, y compris toujours le bénéficiaire», démontrant le rôle progressivement central de la cocréation dans S-D logic. Alors que certains auteurs limitent la portée de la cocréation aux interactions interhumaines (Grönroos et Voima, 2013), d'autres - y compris ceux qui adoptent une perspective de S-D logic - l'étendent pour y incorporer d'autres formes interactives (par exemple, homme-machine; Caic et al., 2018; Brodie et al., 2016), telle qu'adoptée ici.

La cocréation comprend deux composantes: la co et la création. Premièrement, pour témoigner de son interactivité inhérente, son préfixe «co» (avec) dénote la dépendance du concept sur la présence ou les interdépendances avec d'autres acteurs, avec lesquels l'acteur focal interagit, collabore, ou communique (Lusch et al., 2007; Brodie et al., 2013; Chen et al., 2018). La cocréation est donc hautement contextuelle, s'apparentant à la valeur en contexte (Chandler et Vargo, 2011). Deuxièmement, sa partie création révèle la nature proactive, basée sur l'action ou le «faire» du concept (McColl-Kennedy et al., 2012; Cova et al., 2011; Delpechitre et al., 2018; Oertzen et al., 2018).

Contrairement à la cocréation, qui reflète le processus de création de valeur interactive des acteurs, la codestruction reflète l'attrition de valeur par les interactions (Heidenreich et al., 2015; Smith, 2013; Zhang et al., 2018). Conformément à Bowden et al. (2017), les niveaux de cocréation/destruction perçus par les consommateurs peuvent fluctuer dans ou entre les interactions. Ainsi, bien qu'une interaction puisse être considérée comme une valeur ajoutée, une autre peut éroder la valeur perçue (par exemple, un échec du service ; Plé et Chumpitaz Cáceres, 2010). De plus, si des aspects particuliers liés à l'interaction peuvent cocréer de la valeur (par exemple, des temps de réponse rapides de l'entreprise), d'autres peuvent nuire à la valeur (par exemple, la présence d'acteurs détestés dans le service; Clark et al., 2020). À l'instar du compromis de valeur perçu par Zeithaml (1988), la cocréation nette apparait comme l'équilibre de la cocréation/destruction perçue par le consommateur dans une interaction, une collaboration, ou une communication.

Non seulement les participants à l'interaction affectent la cocréation perçue par l'acteur, mais d'autres acteurs en réseau peuvent également exercer des effets indirects, consciemment ou non 
(Leclercq et al., 2016; Ranjan et Read, 2016). Par exemple, les actions d'un serveur robotique ne sont pas seulement affectées par le client présent, mais aussi par les besoins et les désirs antérieurs des clients qui ont contribué à le «former» (Bèzes, 2019; CW, 2020). La cocréation incorpore donc des acteurs individuels et des facteurs plus systémiques qui s'influencent mutuellement (Edvardsson et al., 2011; McColl-Kennedy et al., 2012). Dans cet article, nous nous concentrons sur la valeur cocréée perçue par le consommateur (Busser et Shulga, 2018; Witell et al., 2011). Lusch et collègues (2007) postulent que: «La valeur ne peut être déterminée que par l'utilisateur [c'est-à-dire, consommateur] dans le processus de consommation», justifiant l'importance de la valeur cocréée par le consommateur. Les principaux acteurs avec lesquels les consommateurs peuvent cocréer de la valeur comprennent les entreprises/marques, le personnel de service, et les autres consommateurs (Hult et al., 2011; Clark et al., 2020).

\section{Valeur cocréée}

Malgré les deux décennies de recherche sur la cocréation, la compréhension de son résultat basé sur la valeur de la valeur cocréée est à la traîne (Black et Gallan, 2015; Go Jefferies et al., 2019). Tout comme la cocréation, la valeur cocréée trouve ses origines à la fois dans (par exemple, Cova et Salle, 2008; Cabiddu et al., 2013) et en dehors de S-D logic (par exemple, Prahalad et Krishnan, 2008). Par conséquent, un consensus autour de la définition fait défaut et certains auteurs préfèrent laisser le concept indéfini (par exemple, Go Jefferies et al., 2019). Busser et Shulga (2018, p. 69) définissent la valeur cocréée comme «l'évaluation personnelle d'un consommateur de la signification d'un service basée sur ce qui est apporté et ce qui est réalisé grâce à la collaboration». Nous sommes d'accord sur le caractère hautement subjectif et collaboratif de la valeur cocréée (Vargo et Lusch, 2016; Lee et al., 2012), auquel nous ajoutons le rôle clé habilitant de l'interactivité ou de la communication (Verma et al., 2012; Gummesson et Mele, 2010) ), comme souligné. Nous sommes également d'accord concernant le compromis interne coût/bénéfice des consommateurs dans l'évaluation de leur valeur cocréée (Zeithaml, 1988), qui peut être positive (c'est-à-dire, valeur cocréée) ou négative (c'est-à-dire, valeur codétruite; Heidenreich et al., 2015; Caic et al., 2018).

Black et Gallan (2015, p. 2) considèrent la valeur cocréée comme une valeur perçue par le consommateur grâce à la synergie des «contributions des utilisateurs, du soutien du réseau et des conditions macro-environnementales». Nous convenons que la valeur cocréée par le consommateur est influencée par des forces externes, largement incontrôlables de l'écosystème (par exemple, le marché) qui s'étendent au-delà des seuls participants à l'interaction, comme indiqué pour la cocréation. Autrement dit, la valeur cocréée repose sur un ensemble d'acteurs au niveau micro, méso ou, macro (par rapport à un seul acteur), que ce soit à travers des interactions réelles ou des interdépendances d'acteurs (Akaka et al., 2012). Ici, les acteurs peuvent avoir des objectifs ou des 
programmes différents, ce qui complique potentiellement l'atteinte de la valeur cocréée mutuelle (Hult et al., 2011; Clark et al., 2020). Par exemple, si la réalisation de l'objectif d'un acteur peut cocréer de la valeur pour elle (par exemple, en remportant un concours), elle peut nuire à la valeur d'un autre (Huber et al., 2017; Bailey et al., 2018). Cet exemple illustre également l'importance des objectifs des acteurs dans la promotion de la valeur cocréée (Go Jefferies et al., 2019). Dans l'ensemble, les définitions de Busser et Shulga (2018) et de Black et Gallan (2015) concordent en termes d'importance subjectivement déterminée inhérente à la valeur cocréée pour le consommateur.

Bien que nous distinguions cocréation et valeur cocréée, nous observons un degré de confusion sémantique qui entoure les deux concepts. Autrement dit, comme certains auteurs de cocréation se réfèrent en fait à la valeur cocréée, nous considérons également leurs conceptualisations dans la définition de CDCV. Par exemple, McColl-Kennedy et al. (2012, p. 370) considèrent la cocréation de la valeur comme «l'avantage tiré de l'intégration des ressources grâce aux activités et aux interactions avec les collaborateurs du réseau de services [de l'acteur]». Compte tenu de l'accent mis sur l'avantage de cette définition (c'est-à-dire, sur les résultats basés sur la valeur), le concept central des auteurs révèle en fait une valeur cocréée (par rapport à la cocréation). De même, $\mathrm{Ng}$ et al. (2011, p. 14) définissent la cocréation de valeur comme «[la valeur] qui est [...] créée conjointement entre le client et l'entreprise pour générer un bénéfice», faisant ainsi également référence au résultat basé sur la valeur des processus de cocréation. De même, Hollebeek et al. (2019, p. 168) définissent la cocréation comme «la valeur perçue d'un client résultant d'activités interactives, conjointes, ou collaboratives liées à la marque pour/avec les parties prenantes».

En résumé, notre examen révèle la nature hautement subjective et perçue de la valeur cocréée qui émerge des interactions, de la collaboration, ou de la communication du consommateur avec d'autres participants du réseau, y compris les entreprises/marques, les employés, et les autres consommateurs (Vargo et Lusch, 2016; Ng et al., 2011; Ranjan et Read, 2016; Verma et al., 2012). Compte tenu de notre focalisation sur la valeur cocréée digitale, notre champ d'application se limite à la valeur cocréée via des plateformes digitales (Breidbach et al., 2014), comme expliqué plus en détail dans la section suivante. Sur la base de notre examen, nous définissons CDCV comme la valeur perçue par le consommateur qui résulte de l'interaction, de la collaboration, ou de la communication avec ou via des plateformes digitales.

Reconnaissant la valence différente de la CDCV, son champ d'application comprend la valeur perçue positive (c'est-à-dire, cocréée) et négative (c'est-à-dire, codétruite; Echeverri et Skålén, 2011; Gebauer et al., 2013; Daunt et Harris, 2017). Ainsi, contrairement à la CDCV, qui se transforme en valeur perçue positive par un consommateur à partir d'une interaction, la valeur codétruite est negative (par exemple, par le harcèlement sur Internet; Kowalski et al., 2012). Nous approfondissons 
ensuite l'aspect plateforme digitale de notre définition CDCV en proposant une typologie des plateformes digitales.

\section{Développement conceptuel}

\section{Typologie des plateformes digitales}

Les plateformes digitales, qui sont des «points de contact virtuels activés par la technologie et conçus pour fournir un soutien structurel à... l'échange» (Hollebeek, 2019, p. 89), sont d'importants canaux de création d'interactions et de cocréation de valeur (Ramaswamy et Ozcan, 2018; Breidbach et al., 2014), comme indiqué. Bien que certaines plateformes facilitent les interactions en face à face (par exemple, les réunions), nos analyses se limitent aux plateformes digitales qui permettent les interactions, la collaboration, ou la communication avec les consommateurs via des points de contact technologiques (Ramaswamy et Ozcan, 2016; Kannan et Li, 2017; Troisi et al., 2018). Étant donné que les plateformes digitales génèrent de la valeur cocréée de différentes manières, nous développons une typologie de plateformes digitales qui comprend des plateformes homme-homme et hommemachine, comme détaillé ci-dessous.

Les plateformes homme-homme (H2HP) sont des points de contact technologiques qui assurent la médiation des interactions, la collaboration, ou la communication interhumaine. Ces plateformes offrent une interface qui sert d'intermédiaire pour connecter les individus (par exemple, les applications mobiles; Pirrone et al., 2012; Breidbach et Maglio, 2016). Ici, un partenaire d'interaction peut être connu personnellement ou non (Brzozowski et al., 2008). La concentration relationnelle et communicationnelle des H2HP peut faciliter le développement de liens forts ou faibles (Granovetter, 1983). Compte tenu du rôle de médiation des interactions de ces plateformes, les utilisateurs de H2HP peuvent se présenter en fonction de leur véritable identité ou interpréter un autre personnage (par exemple, des avatars; Lovelock, 2017).

Les H2HP logiciels diffèrent du matériel (périphériques) sur lequel ils sont exécutés, qui sont connus sous le nom d'archétypes de plateforme (Hollebeek et al., 2020a). Les exemples H2HP incluent les réseaux sociaux, les communautés en ligne, les sites Internet, les applications mobiles, les jeux sociaux, la messagerie instantanée, et les plateformes de téléphonie en ligne (Raïes et GavardPerret, 2011 ; Kohler et al., 2011; Bernal-Merino, 2016; He and Yan, 2015; Algharabat, 2018).

Les plateformes homme-machine (H2MP) sont des points de contact informatisés qui se connectent avec les utilisateurs (par exemple, les robots de service; Wirtz et al., 2018; Van Doorn et al., 2017). Autrement dit, la technologie basée sur H2MP est le partenaire d'interaction du consommateur, révélant le rôle d'interaction de ces plateformes, où les interactions peuvent être initiées par l'utilisateur ou la plateforme. Bien que ces plateformes puissent remplir un rôle relationnel 
(par exemple, des robots sociaux établissant des liens avec les utilisateurs), elles - contrairement aux H2HP - ne sont pas conçues pour faciliter les interactions d'homme-homme (Marin et al., 2009). Au lieu de cela, les H2MP exécutent des tâches particulières pour (c'est-à-dire, offrent des services aux) consommateurs en améliorant leur efficacité ou leur performance, grâce à l'intelligence artificielle (Huang et Rust, 2020; Lee et Sathikh, 2013). S'appuyant sur Pradeep et al. (2019), nous classons les sous-types H2MP suivants:

Premièrement, les plateformes basées sur l'automatisation des processus robotiques informatisent les processus existants à forte intensité de main-d'œuvre. Ils fonctionnent grâce à une automatisation basée sur des règles, où des algorithmes linéaires sont utilisés pour répondre aux requêtes de base via des signaux basés sur des capteurs (Hollebeek et al., 2020b). Par exemple, lors de l'utilisation des menus du centre d'appels ou des télécommandes de télévision, les consommateurs signalent l'activité souhaitée (par exemple, centre d'appels - en appuyant sur «1»pour obtenir les détails de leur compte, «2» pour changer d'adresse, etc.), qui à son tour activent des réponses automatiques prédéterminées particulières. Les plateformes basées sur l'automatisation des processus robotiques reflètent ainsi un faible niveau d'intelligence artificielle qui répond à la demande de données de l'utilisateur, sans apprendre ni adapter ses actions dans le processus (Huang et Rust, 2018; Pradeep et al. 2019).

Deuxièmement, les plateformes basées sur l'apprentissage automatique/en profondeur fonctionnent de manière relativement autonome et ajustent automatiquement leurs actions pour répondre à ou anticiper les besoins des utilisateurs, sans nécessiter d'intervention ou de soutien humain (Mende et al., 2019). Les algorithmes d'apprentissage automatique/en profondeur sousjacents de ces plateformes les aident à faire des prédictions de plus en plus précises des besoins ou des comportements des utilisateurs (Marr, 2018; Chen et Lin, 2014), améliorant ainsi leurs performances au fil du temps. Pour augmenter leur précision prédictive, les H2MP s'appuient sur de grands volumes de données d'entraînement, révélant le rôle crucial du big data (Hollebeek et al., 2020b; Arthur, 2013). Des exemples de ces plateformes comprennent les prédictifs SMS/e-mail, les robots de service (par exemple, les serveurs robotiques/réceptionnistes d'hôtel), les chat-bots, les robots sociaux (par exemple, Pepper utilisé pour rencontrer et saluer les clients), les robots médicaux, les assistants personnels intelligents (par exemple, Watson), les voitures autonomes, et les appareils basés sur l'Internet des objets (par exemple, les appareils ménagers intelligents; Kumar et al., 2016; Chérif et Lemoine, 2018; Wirtz et al., 2018; Ng et Wakenshaw, 2017; Goudey et Bonnin, 2016; Leicht et al., 2018).

Nos types de plateformes digitales peuvent fonctionner indépendamment, mais elles peuvent également être connectés les unes aux autres. Par exemple, une page de réseau social peut 
enregistrer les données de flux de clics d'un consommateur, qu'elle transmet ensuite à un algorithme d'apprentissage automatique basé sur H2MP pour identifier les produits qui intéressent l'utilisateur en fonction de son historique de recherche (Chen et Lien, 2014). Dans ces cas, les caractéristiques de chaque plateforme digitale déployée doivent être prises en compte. Cependant, alors que les interactions de machine à machine inhérentes aux formes intégrées de nos types de plateformes digitales offrent des processus de back-office importants (Schweitzer et al., 2019; Chen et al., 2012), nous les excluons de notre typologie de plateformes digitales car elles sont invisibles pour les utilisateurs. Par exemple, bien que des applications artificiellement intelligentes puissent générer un contenu particulier (par exemple, publicitaire) affiché dans les jeux sociaux, le jeu reste la principale plateforme interactive du consommateur. En tant que telles, les interactions de machine à machine opérant en arrière-plan ne relèvent pas de notre typologie. De plus, les interactions de machine à machine s'écartent de la portée de CDCV, qui implique le «faire» actif du consommateur (McCollKennedy et al., 2012), comme indiqué. Plus loin, nous développerons un cadre de CDCV fondé sur la théorie de la présence sociale.

\section{Cadre conceptuel fondé sur la théorie de la présence sociale et des propositions de CDCV}

La théorie de la présence sociale examine la capacité des plateformes de communication à transmettre des indices sociaux et à générer une intimité et une immédiateté perçues par l'utilisateur, qui diffèrent d'une plateforme à l'autre (Short et al., 1976; Gooch et Watts, 2015). Par conséquent, différentes plateformes digitales affectent différemment la valeur perçue par l'utilisateur (Song et al., 2008). Par exemple, si le courrier électronique révèle généralement des niveaux d'intimité et d'immédiateté inférieurs, ceux-ci ont tendance à être plus élevés pour les plateformes d'appel vidéo qui transmettent des signaux sociaux plus étendus (par exemple, Zoom), améliorant ainsi leur capacité de création de valeur (Nakanishi et al., 2011). Cependant, bien que des chercheurs antérieurs aient exploré l'intimité de la plateforme et/ou l'effet de l'immédiateté sur la valeur perçue par l'utilisateur (par exemple, Song et Hollenbeck, 2015), leurs effets respectifs sur la CDCV interactif restent ténus, comme cela est donc exploré dans cet article. Plus précisément, nous posons que des niveaux plus élevés d'intimité et d'immédiateté perçues de la plateforme digitale ont un impact favorable sur CDCV. Bien que l'intimité et l'immédiateté ont tendance à être optimisées dans les interactions en face à face, nous nous concentrons sur leur manifestation à travers les plateformes digitales (OseiFrimpong et McLean, 2018; Van Doorn et al., 2017) et leur impact respectif sur la CDCV.

Comme indiqué, la théorie de la présence sociale propose que l'intimité et l'immédiateté perçues de la plateforme définissent les évaluations de la plateforme par les utilisateurs (Short et al., 1976; Lombard et Ditton, 1997; Tu, 2000). Premièrement, l'intimité reflète la capacité perçue par l'utilisateur d'une plateforme digitale à susciter la chaleur, la proximité, ou l'appartenance à son 
partenaire d'interaction (Baek et al., 2018; Van Doorn et al., 2017). On pense généralement que l'intimité est fonction de la distance physique des partenaires d'interaction, des sujets de conversation, et de la communication non verbale (Argyle et Dean, 1968; Tu, 2000). Parmi celles-ci, la communication non verbale ou la mesure dans laquelle une plateforme permet l'utilisation d'éléments non parlés en échange (par exemple, contact visuel, sourire, langage corporel; Birdwhistell, 1970), est directement affectée par la plateforme digitale déployée. Cependant, malgré leurs différences, la plupart des plateformes digitales autorisent au minimum l'utilisation du paralangage. Le paralangage fait référence à «des manifestations écrites d'éléments non verbaux... (par exemple, tactiles, visuels, toniques) qui complètent ou remplacent le langage écrit et qui peuvent être exprimées par des mots, des symboles, des images, des signes de ponctuation, des démarcations, ou toute combinaison de ceux-ci» (Luangrath et al. ., 2017, p. 98), y compris les emoji (Hill, 2016). En règle générale, plus la gamme d'indices non verbaux utilisés sur ou via une plateforme digitale est grande, plus son intimité perçue est élevée (Hopkins, 2020).

Deuxièmement, l'immédiateté est la capacité perçue par l'utilisateur d'une plateforme digitale à donner de l'urgence ou de l'importance à un échange (par exemple, par les destinataires qui consultent et répondent rapidement à un message; Wiener et Mehrabian, 1968), qui diffère également d'une plateforme à l'autre. Par exemple, la messagerie instantanée a tendance à recevoir une réponse plus rapide que les formulaires de demande en ligne. En règle générale, les réponses plus rapides favorisent l'immédiateté croissante de la plateforme (Gunawardena et Zittle, 1997). Alors que l'immédiateté comprend deux éléments principaux, dont (a) la capacité de la plateforme à formuler une réponse en temps opportun, et (b) la décision du destinataire concernant le moment de répondre, nous soulignons le premier aspect de l'immédiateté, c'est-à-dire celui basé sur la plateforme digitale. Ici, une condition nécessaire pour une immédiateté élevée est la promotion de la perception par l'utilisateur, d'une efficacité accrue de la plateforme, ou sa fiabilité dans la transmission d'un message ou d'un contenu à son (ses) destinataire(s) (Short et al., 1976; Smedlund et al., 2015). Nous présentons ci-dessous notre model fondé sur la théorie de la présence sociale, qui explore l'effet de l'intimité et de l'immédiateté de la plateforme digitale sur CDCV (voir Fig.1).

\section{Veuillez insérer la Fig.1 à peu près ici}

Nous postulons que la plateforme digitale déployée affecte l'association de l'intimité/immédiateté et la CDCV (Figure 1). Ci-dessous, nous développons des Propositions qui détaillent comment le model propose des associations pour nos plateformes digitales suggérées. Premièrement, l'intimité reflète la capacité perçue par l'utilisateur d'une plateforme à favoriser la proximité avec son partenaire d'interaction (Short et al., 1976), comme indiqué. De plus, les H2HP jouent le rôle d'intermédiaire dans la connexion des utilisateurs, révélant leur rôle principalement 
relationnel ou communicationnel. Cependant, les H2MP aident principalement les utilisateurs à exécuter plus efficacement des tâches particulières (par exemple, les appareils ménagers intelligents), exposant leur nature plus utilitaire (Voss et al., 2003). Nous en déduisons donc que les H2HP actuels sont susceptibles de générer une plus grande intimité perçue par l'utilisateur que les H2MP. Néanmoins, alors que les H2MP basés sur l'apprentissage automatique/en profondeur continuent de se développer, nous prévoyons qu'ils posséderont une capacité relationnelle croissante au fil du temps (par exemple, des assistants personnels virtuels formeront des liens avec leurs utilisateurs; Van Doorn et al., 2017). Nous posons (voir aussi Fig.1):

P1a: Les H2HP contemporains (par rapport aux H2MP) génèrent généralement une plus grande intimité perçue par l'utilisateur, ce qui permet une plus grande contribution du premier à la CDCV à cet égard. Cependant, à mesure que les H2MP basés sur l'apprentissage automatiquelen profondeur se développent pour remplir un rôle de plus en plus relationnel, leur capacité à favoriser l'intimité perçue par l'utilisateur augmentera avec le temps.

Nous comparons ensuite nos sous-catégories H2MP de plateformes basées sur l'automatisation des processus robotiques et l'apprentissage automatique/en profondeur. Alors que ces derniers sont capables «d'apprendre» en ajustant ou en affinant leurs actions pour offrir des suggestions ou des solutions de plus en plus préférées par les utilisateurs, les premiers ne font que répondre aux signaux basés sur les capteurs des utilisateurs, sans évoluer ni apprendre (Pradeep et al., 2019; Huang et Rust, 2018). Donc, les plateformes basées sur l'apprentissage automatique/en profondeur se rapprochent du consommateur, développant ainsi des relations plus intimes (par rapport aux plateformes basées sur l'automatisation des processus robotiques). Nous stipulons (voir également Fig.1):

P1b: Les H2MP basés sur l'apprentissage automatiquelen profondeur (par rapport à l'automatisation des processus robotiques) génèrent généralement une plus grande intimité perçue par l'utilisateur, ce qui permet une plus grande contribution du premier à la CDCV à cet égard.

Deuxièmement, l'immédiateté dénote la capacité perçue d'une plateforme digitale à donner de l'urgence à un échange (Short et al., 1976), dont nous anticipons qu'elle diffère d'une plateforme à une autre. Par exemple, les H2HP délivrent des messages ou du contenu aux utilisateurs de la plateforme et leur permettent d'interagir (Hollebeek et al., 2014). Comme les destinataires peuvent afficher ou répondre aux messages à leur discrétion, les interactions peuvent présenter un délai de réponse (Wu et al., 2014). Les consommateurs peuvent également ignorer les messages ou le contenu publiés sur ces plateformes, ce qui peut entraîner un retard ou une non-réponse et une faible immédiateté (par exemple, dans les communautés en ligne). Cependant, comme les H2MP sont le partenaire d'interaction des utilisateurs (Mende et al., 2019), ils offrent généralement une réponse 
plus rapide que les $\mathrm{H} 2 \mathrm{HP}$, étant donné leur présence en temps réel dans les interactions. Nous postulons (voir aussi Fig.1) :

P2a: Les H2MP (par rapport aux H2HP) génèrent généralement une plus grande immédiateté perçue de la plateforme, ce qui permet une contribution élevée de ces dernières à la CDCV à cet égard.

Nous comparons ensuite l'immédiateté entre nos sous-catégories H2MP. Premièrement, les plateformes basées sur l'automatisation des processus robotiques mécanisent les processus banals à forte intensité de main-d'œuvre existants (Pradeep et al., 2019), comme indiqué. La grande immédiateté de ces plateformes a tendance à être associée à un niveau de précision élevé de leur réponse aux invites de l'utilisateur lors de l'exécution de tâches particulières (Willcocks et al., 2015). Par conséquent, la réponse cohérente et opportune des utilisateurs de ces plateformes peut faciliter leur contribution stable à la $\mathrm{CDCV}$, lorsque les utilisateurs continuent à valoriser régulièrement leurs interactions avec la plateforme au fil du temps. Cependant, l'immédiateté élevée de ces plateformes peut également voir une contribution de moins en moins importante à la CDCV, lorsque que les consommateurs s'habituent de plus en plus à utiliser les fonctionnalités de la plateforme, atténuant ainsi leurs évaluations au fil du temps (Heitz et al., 2019; Hollebeek et al., 2020b). En revanche, l'immédiateté élevée des plateformes basées sur l'apprentissage automatique/en profondeur est sujette à des débuts timides, mais s'améliore progressivement au fil du temps (Pradeep et al., 2019), ce qui donne une contribution de plus en plus positive à la CDCV. Nous formulons (voir également Fig.1):

P2b: La grande immédiateté des plateformes basées sur l'automatisation des processus robotiques contribue généralement de manière stable ou en baisse à la CDCV. Cependant, l'immédiateté élevée des plateformes basées sur l'apprentissage automatiquelen profondeur est susceptible d'apporter une contribution croissante à la CDCV lors d'interactions répétées.

\section{Discussion and implications}

Nous discutons ensuite les implications importantes qui découlent de nos analyses, suivies d'un aperçu des limites de cette recherche, à partir de laquelle nous développons des pistes pour une enquête plus approfondie.

\section{Implications théoriques}

Nous proposons d'abord des observations générales sur l'état actuel de la recherche sur la valeur cocréée et ses répercussions générales sur le domaine, suivies d'une discussion sur les implications spécifiques qui découlent de notre model et des Propositions de CDCV.

Implications théoriques générales. Nous proposons les réflexions suivantes sur l'état actuel de la recherche sur la valeur cocréée. Premièrement, les disparités de perception concernant la conceptualisation de la valeur cocréée et la gamme croissante de concepts liés à la valeur cocréée justifient une attention académique. Par exemple, les chercheurs explorent des concepts tels que la 
valeur cocréée, les marques cocréées, le capital de marque cocréé, l'expérience cocréée, les connaissances cocréées, la responsabilité sociale cocréée, la récupération de services cocréée, la sensibilisation à la santé cocréée, etc. (Gyrd-Jones et Kornum, 2013 ; Kull et Heath, 2016 ; Helm et Jones, 2010 ; Mahr et al., 2014; Mossberg et al., 2014; Dong et al., 2008). Cependant, le déploiement de ces concepts, qui, quoique différents, désignent souvent des idées très similaires, risque de développer une vision fragmentée et floue (Ranjan et Read, 2016; Hollebeek et al., 2019). C'est pourquoi il nous paraît nécessaire que soit menée une recherche rigoureuse sur la consolidation de valeur cocrée, et qui en fasse l'inventaire puis identifie les caractéristiques généralisables de ce concept.

Deuxièmement, la compréhension du réseau nomologique de la valeur cocréée est de plus en plus floue. Les auteurs ont utilisé des prismes théoriques différents pour explorer la valeur cocréée, y compris S-D logic (Vargo et Lusch, 2010), la théorie de l'échange social (Grace et Iacono, 2015), ou la théorie de la motivation (Chou et Chen, 2018), qui proposent généralement des jeux uniques d'antécédents et de conséquences de la valeur cocréée. Ce qui est donc nécessaire, c'est une recherche plus approfondie qui unifie la la perspective de la valeur cocréée pour l'ensemble des points de vues théoriques (Saha et al., 2020).

Troisièmement, nous avons développé un cadre fondé sur la théorie de la présence sociale et des Propositions de CDCV, qui mettent en évidence l'effet favorable de l'intimité et de l'immédiateté perçues par la plateforme digitale sur la CDCV. Autrement dit, les plateformes digitales qui optimisent l'intimité et l'immédiateté perçues par l'utilisateur stimulent principalement la CDCV. Cependant, de futures recherches empiriques sont nécessaires pour tester et valider nos résultats conceptuels. Ci-dessous, nous détaillons les implications spécifiques de notre model et des Propositions de CDCV.

Implications découlant de notre model et Propositions de CDCV. Notre model et nos Propositions avancent un aperçu de la CDCV, qui reste pour autant floue à ce jour (Huber et al., 2017; Kristensson et al., 2008; Song et al., 2008). Ci-dessous, nous détaillons les principales implications qui émergent de nos résultats, telles que structurées par nos Propositions (veuillez également vous référer aux questions de recherche spécifiques énumérées dans le Tableau 1).

\section{Veuillez insérer le Tableau 1 à peu près ici}

Premièrement, P1a se lit comme suit: «Les H2HP contemporains (par rapport aux H2MP) génèrent généralement une plus grande intimité perçue par l'utilisateur, ce qui permet une plus grande contribution du premier à la CDCV à cet égard. Cependant, à mesure que les H2MP basés 
sur l'apprentissage automatiquelen profondeur se développent pour remplir un rôle de plus en plus relationnel, leur capacité à favoriser l'intimité perçue par l'utilisateur augmentera avec le temps.» Dans la théorie de la présence sociale, la capacité d'une plateforme à générer une intimité perçue par l'utilisateur est essentielle (Short et al., 1976; Osei-Frimpong et McLean, 2018). Nous prévoyons une plus grande capacité des H2HP contemporains (par rapport aux H2MP) à générer une intimité et CDCV perçue par le consommateur, comme indiqué. Toutefois, les développements continus de l'intelligence artificielle et l'acceptation croissante de ces solutions par les consommateurs réduiront l'écart au fil du temps. À l'avenir, il est donc intéressant d'explorer les meilleures pratiques de conception H2MP (par exemple, de quelle manière ces plateformes devraient-elles se rapprocher (par rapport à s'en écarter) du comportement humain en situation d'interrelation?; Kim et al., 2019).

En comparant nos sous-catégories H2MP, P1b déclare: «Les H2MP basés sur l'apprentissage automatiquelen profondeur (par rapport à l'automatisation des processus robotiques) génèrent généralement une plus grande intimité perçue par l'utilisateur, ce qui permet une plus grande contribution du premier à la CDCV à cet égard.» Les plateformes basées sur l'automatisation des processus robotiques sont principalement utilisées pour informatiser les tâches laborieuses (Pradeep et al., 2019). En raison de leur orientation utilitaire, la capacité de ces plateformes à générer une intimité perçue par l'utilisateur, et donc CDCV, est limitée. Cependant, équipées de la capacité d'interpréter les signaux (non) verbaux des consommateurs et de prédire les besoins des utilisateurs, les plateformes basées sur l'apprentissage automatique/en profondeur proposent des solutions jugées souhaitables par les utilisateurs (Huang et Rust, 2020), favorisant ainsi un niveau d'intimité (par exemple, via des offres personnalisées). Cependant, une meilleure compréhension est nécessaire concernant la capacité respective de ces plateformes à générer une intimité/immédiateté et CDCV perçue par l'utilisateur. Par exemple, comment les algorithmes d'apprentissage automatique/en profondeur devraient-ils être programmés pour améliorer l'intimité de la plateforme?

P2a postule : «Les H2MP (par rapport aux H2HP) génèrent généralement une plus grande immédiateté perçue de la plateforme, ce qui permet une contribution élevée du premier à la CDCV à cet égard.» $\mathrm{Au}$ fur et à mesure que les H2HP connectent les acteurs humains, leur réponse à l'expéditeur peut être retardée, bien que les H2MP aient tendance à offrir un retour utilisateur plus immédiat (Pradeep et al., 2019). Donc, les H2MP (par rapport aux H2HPs) contribuent davantage à la CDCV. Cependant, ces affirmations nécessitent des tests empiriques futurs. Par exemple, comment les interactions H2HP qui présentent une réponse immédiate se comparent-elles aux interactions $\mathrm{H} 2 \mathrm{MP}$ en termes de CDCV respectifs ? Quelle est l'importance relative de l'immédiateté d'une 
plateforme digitale par rapport à sa capacité à générer une intimité perçue par l'utilisateur, ou (comment) ces facteurs peuvent-ils interagir pour conduire la CDCV?

P2b déclare: «La grande immédiateté des plateformes basées sur l'automatisation des processus robotiques contribue généralement de manière stable ou en baisse à la CDCV. Cependant, l'immédiateté élevée des plateformes basées sur l'apprentissage automatiquelen profondeur est susceptible d'apporter une contribution croissante à la CDCV lors d'interactions répétées.» Comme indiqué, les plateformes basées sur l'automatisation des processus robotiques mécanisent des tâches relativement simples. En revanche, les plateformes basées sur l'apprentissage automatique/en profondeur peuvent être utilisées pour prédire ou anticiper les besoins des consommateurs (Huang et Rust, 2018). Par conséquent, à mesure que les consommateurs s'habituent à interagir avec des plateformes basées sur l'automatisation des processus robotiques, CDCV a tendance à rester stable ou à diminuer avec le temps, comme indiqué. Cependant, à mesure que la précision prédictive des plateformes basées sur l'apprentissage automatique/en profondeur s'améliore au fil des interactions répétées (Mende et al., 2019), ces plateformes apportent une contribution croissante à la CDCV. Les questions qui subsistent sont les suivantes: Quelles fonctionnalités d'apprentissage automatique/en profondeur sont particulièrement propices à la stimulation de la CDCV? Quelles tactiques les plateformes basées sur l'automatisation des processus robotiques peuvent-elles déployer pour arrêter ou ralentir l'attrition de CDCV? Nous appliquons ensuite nos Propositions à la pratique du marketing pour illustrer leur applicabilité pratique.

\section{Implications pratiques}

En plus de ses implications théoriques, cette recherche génère également des implications notables pour la conception et la mise en œuvre de plateformes digitales, comme indiqué ci-dessous. Premièrement, P1a se lit comme suit : «Les H2HP contemporains (par rapport aux H2MP) génèrent généralement une plus grande intimité perçue par l'utilisateur, ce qui permet une plus grande contribution du premier à la CDCV à cet égard. Cependant, à mesure que les H2MP basés sur l'apprentissage automatiquelen profondeur se développent pour remplir un rôle de plus en plus relationnel, leur capacité à favoriser l'intimité perçue par l'utilisateur augmentera avec le temps.» Les managers doivent comprendre dans quelle mesure et dans quelles occasions les consommateurs souhaitent nouer des liens étroits avec leur partenaire d'interaction (Gummesson, 2011). Dans ces cas, nous vous recommandons d'adopter principalement les H2HP pour optimiser la CDCV, étant donné le rôle relationnel principal de connexion des utilisateurs de ces plateformes. Cependant, les contextes nécessitant des interactions plus éloignées sont mieux servis par des H2MP moins intimes (par exemple, les plateformes basées sur l'automatisation des processus robotiques). Cependant, comme le déclare P1a, nous prévoyons que l'avantage relationnel relatif des H2HP (par 
rapport aux H2MP) diminuera à mesure que la capacité relationnelle des H2MP basés sur l'apprentissage automatique/en profondeur continuera de se développer au fil du temps (Pradeep et al., 2019), augmentant leur capacité à favoriser les liens étroits avec les utilisateurs.

Deuxièmement, P1b postule: «Les H2MP basés sur l'apprentissage automatiquelen profondeur (par rapport à l'automatisation des processus robotiques) génèrent généralement une plus grande intimité perçue par l'utilisateur, ce qui permet une plus grande contribution du premier à la CDCV à cet égard.» Alors que les plateformes basées sur l'automatisation des processus robotiques informatisent les tâches de routine, elles n'évoluent pas et n'ajuste pas leurs actions en fonction des commentaires des utilisateurs, comme indiqué. Cependant, étant donné leur capacité à apprendre, les plateformes basées sur l'apprentissage automatique/en profondeur régulent leur comportement de manière autonome pour améliorer les résultats des utilisateurs (Huang et Rust, 2020). Pour optimiser la CDCV, nous recommandons donc les dernières plateformes (par rapport aux anciennes), en particulier parce que la capacité de génération de CDCV des plateformes basées sur l'automatisation des processus robotiques peut également diminuer avec le temps (voir P2b). Comme indiqué, ces plateformes peuvent également être déployées ensemble (par exemple, en utilisant des plateformes basées sur l'automatisation des processus robotiques pour faciliter les tâches de bas niveau, qui activent des applications artificiellement intelligentes qui soient pertinentes; Pradeep et al., 2019).

Troisièmement, P2a propose: «Les H2MP (par rapport aux H2HP) génèrent généralement une plus grande immédiateté perçue de la plateforme, ce qui permet une contribution élevée du premier à la CDCV à cet égard.» Les H2HP peuvent entraîner une réponse retardée, car les utilisateurs peuvent accéder aux messages ou au contenu et y répondre à leur rythme, comme indiqué. Cependant, pour les H2MP, l'immédiateté de la réponse de la plateforme - associée à sa précision est déterminante dans les évaluations des utilisateurs. Par conséquent, dans les contextes exigeant une urgence élevée, des plateformes très immédiates offrant un retour instantané (par exemple, le chat en direct) devraient être utilisées (Lv et al., 2018). Cependant, des occasions moins urgentes (par exemple, les processus d'examen par les pairs) peuvent entraîner l'adoption de plateformes moins immédiates (par exemple, le courrier électronique), ce qui peut entraîner un coût moindre (Rice, 1993).

Enfin, P2b déclare: «La grande immédiateté des plateformes basées sur l'automatisation des processus robotiques contribue généralement de manière stable ou en baisse à la CDCV. Cependant, l'immédiateté élevée des plateformes basées sur l'apprentissage automatiquelen profondeur est susceptible d'apporter une contribution croissante à la CDCV lors d'interactions répétées.» Les évaluations stables par les utilisateurs des plateformes basées sur l'automatisation des 
processus robotiques auront tendance à apporter une contribution relativement faible à la CDCV, en raison de la nature banale de leurs tâches (Hollebeek et al., 2020b). Cependant, les évaluations des consommateurs de ces plateformes peuvent également décliner (par exemple, à mesure qu'ils s'habituent à utiliser ces plateformes; Heitz et al., 2019). En revanche, grâce à leur capacité à apprendre, les plateformes basées sur l'apprentissage automatique/en profondeur ont tendance à apporter une contribution croissante à la $\mathrm{CDCV}$, comme indiqué. Pourtant, à titre de mise en garde, le principe du garbage in, garbage out (GIGO) s'applique à ces dernières plateformes (Weyerer et Langer, 2019). Autrement dit, l'utilisation de données d'apprentissage de mauvaise qualité entraîne une détérioration (par rapport à l'amélioration) des performances de ces plateformes, ce qui réduit la CDCV ou la transforme en valeur codétruite. Par conséquent, nous recommandons d'utiliser les H2MP (par rapport aux H2HP) pour optimiser l'immédiateté de la plateforme, à condition que leurs solutions offrent une précision de réponse élevée. Pour garantir leur précision élevée (et croissante), ces plateformes nécessitent en permanence de grands volumes de données d'apprentissage de haute qualité (Pradeep et al., 2019).

\section{Limitations et recherches complémentaires}

Cette recherche a plusieurs limites qui offrent des opportunités pour une étude plus approfondie. Premièrement, la nature conceptuelle du cadre nécessite de futurs tests empiriques (Yadav, 2010). Dans cette veine, il est intéressant de mieux comprendre la contribution relative de nos antécédents identifiés et fondés sur la théorie de la présence sociale à la CDCV. Par exemple, si l'intimité perçue d'une plateforme digitale est identifiée comme un moteur CDCV primordial, alors son développement (par rapport à celui de l'immédiateté) doit être priorisé. En outre, comment l'intimité et l'immédiateté de la plateforme digitale perçues par le consommateur peuvent-elles être encouragées pour augmenter la CDCV? Quelles conséquences la CDCV peut-elle générer? De plus, bien que nous abordions la CDCV perçue par le consommateur, les études futures pourraient adopter une perspective bilatérale (par exemple, consommateur / entreprise) ou plus large de la valeur digitale cocréée (Lenka et al., 2016; Alexander et al., 2018), y compris en quantifiant la valeur asymétrique probable de plusieurs acteurs qui découle des interactions digitales (Edvardsson et al., 2011; Clark et al., 2020).

Deuxièmement, notre typologie de plateformes digitales nécessite une validation empirique. Par exemple, des expériences futures peuvent manipuler des principes clés H2HP/H2MP pour en tirer des informations supplémentaires (par exemple, attributs relationnels/fonctionnels étendus ou limités ; Fisher, 1971). Compte tenu de leur nouveauté et de leur évolution rapide, les H2MP en particulier méritent des recherches supplémentaires (Mende et al., 2019). Par exemple, nous nous attendons à voir l'intégration croissante de fonctionnalités utilitaires et plus sociales (par exemple, 
compagnonnage) dans les futurs H2MP, comme discuté. Nous anticipons ainsi la capacité relationnelle croissante de ces plateformes, conduisant les consommateurs à développer un attachement croissant à ces plateformes, notamment dans certaines cultures (Kitano, 2006; Hollebeek, 2018), ce qui mérite une étude plus approfondie. Par conséquent, les H2MP progressivement relationnels sont mieux perçus sur un continuum fonctionnel à relationnel. Malgré ces développements, nous nous attendons à ce que notre typologie de plateforme digitale se maintienne, car les H2MP - tout en remplissant un rôle relationnel potentiel - ne sont pas conçus pour permettre des interactions homme-homme, comme les H2HP (Marin et al., 2009). Compte tenu du besoin inné d'appartenance de l'espèce humaine (Maslow, 1954), nous nous attendons à un désir continu d'interactions basées sur H2HP, bien que leur nature puisse changer avec le temps. Voici quelques exemples de questions à examiner: Quelles plateformes digitales sont les plus propices à l'optimisation de la CDCV, tout en minimisant la valeur détruite par le codage numérique ? Comment la fonctionnalité de plus en plus relationnelle des H2MP affectera-t-elle CDCV?

Troisièmement, il est intéressant d'explorer les associations théoriques proposées dans la Figure 1 pour les consommateurs utilisant plusieurs plateformes digitales (Uhrich, 2014). Par exemple, quelles plateformes les consommateurs utilisent-ils généralement ensemble, comment les utilisateurs perçoivent-ils l'intimité et l'immédiateté des différentes plateformes à travers les segments de clientèle, et comment l'utilisation de la plateforme intégrative des consommateurs affecte-t-elle CDCV? Par conséquent, dans quelle mesure les H2HP et H2MP devraient-elles être conçues conjointement pour optimiser la CDCV pour les utilisateurs multi-plateformes (Tixier et al., 2010; Belboula et al., 2018)? Comment la conception H2HP/H2MP peut-elle stimuler les changements de valence CDCV négatifs à positifs, tout en minimisant les oscillations inverses (Bowden et al., 2017)?

Quatrièmement, nous encourageons des recherches plus poussées qui étudient la CDCV au sein de réseaux nomologiques plus larges, alternatifs, ou unificateurs, qui peuvent être comparées à nos analyses fondées sur la théorie de la présence sociale (Short et al., 1976; Osei-Frimpong et McLean, 2018). Ainsi, bien que la théorie de la présence sociale offre un prisme approprié pour explorer la CDCV à travers les plateformes (digitales), des alternatives existent (par exemple, la théorie de l'échange social ou de la motivation), comme discuté. Ces futures découvertes pourraient être comparées ou intégrées aux nôtres (par exemple pour compléter ou affiner notre vision). 


\section{Fig.1: Cadre conceptuel}

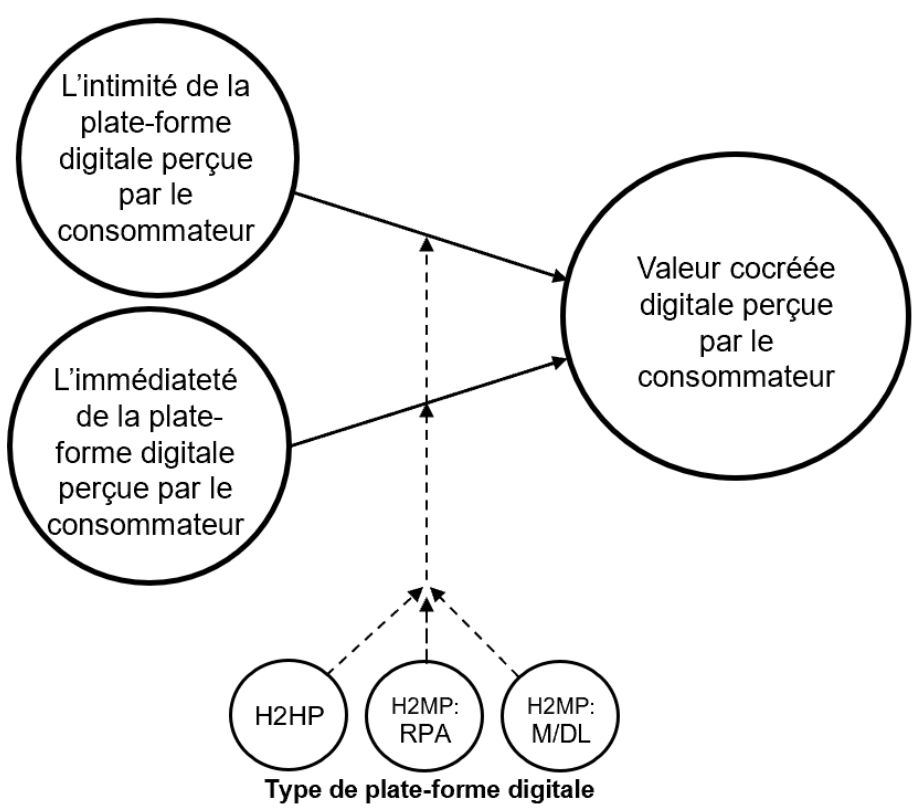

Notes - H2HP: Plateforme homme-homme; H2MP: Plateforme homme-machine; RPA: Plateforme basée sur l'automatisation des processus robotiques; M/DL: Plateforme basée sur l'apprentissage automatique/en profondeur. 


\section{Tableau 1: Exemples de questions de recherche}

\section{Proposition}

P1a: Les H2HP contemporains (par rapport aux H2MP) génèrent généralement une plus grande intimité perçue par l'utilisateur, ce qui permet une plus grande contribution du premier à la CDCV à cet égard. Cependant, à mesure que les H2MP basés sur l'apprentissage automatique/en profondeur se développent pou remplir un rôle de plus en plus relationnel, leur capacité à favoriser l'intimité perçue par l'utilisateur augmentera avec le temps.

P1b: Les H2MP basés sur l'apprentissage automatique/en profondeur (par rapport à l'automatisation des processus robotiques) génèrent généralement une plus grande intimité perçue par l'utilisateur, ce qui permet une plus grande contribution du premier à la CDCV à cet égard.

P2a: Les H2MP (par rapport aux H2HP) génèrent généralemen une plus grande immédiateté perçue de la plateforme, ce qui permet une contribution élevée du premier à la $\mathrm{CDCV}$ à cet égard.

P2b: La grande immédiateté des plateformes basées sur l'automatisation des processus robotiques contribue généralement de manière stable ou en baisse à la CDCV. Cependant, l'immédiateté élevée des plateformes basées sur l'apprentissage automatique/en profondeur est susceptible d'apporter une contribution croissante à la CDCV lor d'interactions répétées.

\section{Exemples de questions de recherche}

Quel est l'effet de l'intimité de la plateforme digitale sur la CDCV pour des H2HP et H2MP particuliers (OseiFrimpong \& McLean, 2018)?

- Comment l'intimité perçue d'une plateforme digitale affecte-t-elle la valeur cocréée (ou codétruite) avec les entreprises par rapport aux autres consommateurs?

Quelle est la contribution relative de l'intimité d'une plateforme à la CDCV?

- L'intimité et l'immédiateté interagissent-elles pour affecter la CDCV dans des contextes digitaux particuliers (Hair et al., 2018)?

- Comment l'utilisation multiple des plateformes digitales par les consommateurs affecte-t-elle l'effet individuel/conjoint de chaque plateforme sur la CDCV (Larivière et al., 2017)?

- Quelle est la meilleure composition de plateformes digitales pour optimiser l'intimité de la plateforme perçue par le consommateur?

Comment les attributs H2HP/H2MP spécifiques (par exemple, relationnels/fonctionnels) affectent-ils la CDCV?

Quel est l'effet de l'immédiateté perçue d'une plateforme digitale sur la CDCV à travers les types de plateforme (Li et al., 2018)?

- Comment l'immédiateté perçue d'un H2MP (par rapport à H2HP) influence-t-elle la valeur cocréée/codétruite avec les entreprises ou les consommateurs?

- Quels sont les principaux attributs H2HP/H2MP qui contribuent à l'immédiateté de la plateforme et comment affectent-ils CDCV?

- Comment le rôle relationnel croissant des H2MP va-t-il progressivement compléter ou concurrencer les H2HPs?

- Dans quelle mesure les plateformes basées sur l'automatisation des processus robotiques et l'apprentissage automatique/en profondeur débloquent-elles respectivement la CDCV et la valeur codétruite (Smith, 2013)?

- Si les consommateurs utilisent plusieurs plateformes digitales, (comment) cela affecte-t-il la contribution respective de chaque plateforme à la CDCV?

Comment la contribution décroissante des H2HPs à la CDCV peut-elle être minimisée ou inversée (Hollebeek et al., 2020b)? 


\section{Références}

Akaka, M., Vargo, S., et Lusch, R. (2012), An exploration of networks in value co-creation: A service-ecosystems view, Review of Marketing Research, 9: 13-50.

Alexander, M., Jaakkola, E., et Hollebeek, L. (2018), Zooming out: Actor engagement beyond the dyadic, Journal of Service Management, 29(3): 333-351.

Algharabat, R. (2018), The role of telepresence and user engagement in cocreation value and purchase intention: Online retail context, Journal of Internet Commerce, 17(1): 1-25.

Alves, H., Fernandes, C., et Raposo, M. (2016), Value co-creation: Concept and contexts of application and study, Journal of Business Research, 69(5): 1626-1633.

Anderson, J. (1995), Relationships in business markets: Exchange episodes, value creation, and their empirical assessment. Journal of the Academy of Marketing Science, 23(4): 346-350.

Argyle, M., Lalljee, M., et Cook, M. (1968), The effects of visibility on interaction in the dyad, Human Relations, 21: 3-17.

Arthur, L. (2013), Big Data Marketing, Wiley.

Baek, E., Choo, H., et Lee, S. (2018), Using warmth as the visual design of a store: Intimacy, relational needs, and approach intentions, Journal of Business Research, 88: 91-101.

Bailey, D., Pitelis, C., et Tomlinson, P. (2018), A place-based developmental regional industrial strategy for sustainable capture of co-created value. Cambridge Journal of Economics, 42(6): 1521-1522.

Balaji, M. et Roy, S. (2017), Value co-creation with Internet of Things technology in the retail industry, Journal of Marketing Management, 33(1-2): 7-31.

Banker, R., Mitra, S., et Sambamurthy, V. (2011), The effects of digital trading platforms on commodity prices in agricultural supply chains, MIS Quarterly, 35(3): 599-611.

Belboula, I., Ackermann, C., et Mathieu, J. (2018), Product design and hierarchized persuasion process: An application to three household electrical products, Recherche et Applications en Marketing, 33(4): 2-23.

Bernal-Merino, M. (2016), Glocalization and co-creation: Trends in international game production. In: Esser, A., Smith, I., et Bernal-Merino, M. (Eds.), Media Across Borders, Routledge.

Bèzes, C. (2019), What kind of in-store smart retailing for an omnichannel real-life experience? Recherche et Applications en Marketing, 34(1): 91-112.

Birdwhistell, R. (1970), Kinesics and Context, Philadelphia: University of Philadelphia Press.

Black, H. et Gallan, S. (2015), Transformative service networks: Cocreated value as well-being, Service Industries Journal, 35(15-16): 826-845.

Bolton, R. et Saxena-Iyer, S. (2009), Interactive services: A framework, synthesis and research directions," Journal of Interactive Marketing, 23(1): 91-104.

Bowden, J., Conduit, J., Luoma-Aho, V., Hollebeek, L., et Solem, B. (2017), Engagement valence duality and spillover effects in online brand communities. Journal of Service Theory and Practice, 27(4): 877-897.

Breidbach, C., Brodie, R., et Hollebeek, L. (2014), Beyond virtuality: From engagement platforms to engagement ecosystems, Managing Service Quality, 24(6): 592-611.

Breidbach, C. et Maglio, P. (2016), Technology-enabled value co-creation: An empirical analysis of actors, resources, and practices, Industrial Marketing Management, 56: 73-85.

Brodie, R., Ilic, A., Juric, B. et Hollebeek, L. (2013), Consumer engagement in a virtual brand community: An exploratory analysis, Journal of Business Research, 66(1): 105-114.

Brodie, R., Jaakkkola, E., Fehrer, J., Hollebeek, L., et Conduit, J. (2016), From customer to actor engagement: Exploring a broadened domain. EMAC, Oslo.

Brzozowski, M., Hogg, T., et Szabo, G. (2008), Friends and foes: Ideological social networking, SIGCHI Conference: Human Factors in Computing Systems, 817-820.

Buonincontri, P., Morvillo, A., Okumus, F. et Van Niekerk, M. (2017), Managing the experience co-creation process in tourism destinations: Empirical findings from Naples, Tourism Management, 62(octobre): 264-277.

Busser, J. et Shulga, L. (2018), Co-created value: Multidimensional scale and nomological network, Tourism Management, 65(avril): 69-86.

Cabiddu, F., Lui, T., et Piccoli, G. (2013), Managing value co-creation in the tourism industry, Annals of Tourism Research, 42(juillet): 86-107.

Caic, M., Odekerken-Schröder, G., et Mahr, D. (2018), Service robots: Value co-creation and co-destruction in elderly care networks, Journal of Service Management, 29(2): 178-205.

Chandler, J. et Vargo, S. (2011), Contextualization and value-in-context: How context frames exchange, Marketing Theory, 11(1): 35-49.

Chen, K. et Lien, S. (2014), Machine-to-machine communications: Technologies and challenges, Ad Hoc Networks, 18(juillet): 3-23.

Chen, M., Wan, J., et Li, F. (2012), Machine-to-machine communication: Architectures, standards, and applications, KSII Transactions on Internet and Information Systems, 6(2): 480-497. 
Chen, T., Drennan, J., Andrews, L., et Hollebeek, L. (2018), User experience sharing: Understanding customer initiation of value co-creation in online communities, European Journal of Marketing, 52(5/6): 1154-1184.

Chen, X. et Lin, X. (2014), Big data deep learning: Challenges and perspectives, IEEE Xplore. Accédé: https://ieeexplore.iee.org/abstract/document/6817512.

Chérif, E. et Lemoine, J-F. (2018), Anthropomorphic virtual assistants and the reactions of internet users: An experiment on the assistant's voice, Recherche et Applications en Marketing, 34(1): 28-47.

Chou, S. et Chen, Y. (2018), The impact of money and task complexity on consumer co-creation intention, Marketing Review, 15(1): 67-100.

Chumpitaz Cáceres, R., et Paparoidamis, N. (2007), Service quality, relationship satisfaction, trust, commitment and business-to-business loyalty, European Journal of Marketing, 41(7/8): 836-867.

Clark, M., Lages, R., et Hollebeek, L. (2020), Friend or foe? Customer engagement's value-based effects on fellow customers and the firm, Journal of Business Research, Dans la presse, DOI: https://doi.org/10.1016/j.jbusres.2020.03.011.

Cova, B., Dalli, D., et Zwick, D. (2011), Critical perspectives on consumers' role as producers: Broadening the debate on value co-creation in marketing processes, Marketing Theory, 11(3): 231-241.

Cova, B. et Salle, R. (2008), Marketing solutions in accordance with the S-D logic: Co-creating value with customer network actors, Industrial Marketing Management, 37(3): 270-277.

CW (2020), Citation Withheld, Cocreation Document de travail.

Dahl, A., Milne, G., et Peltier, J. (2019), Digital health information seeking in an omni-channel environment: A shared decision-making and service-dominant logic perspective, Journal of Business Research, Dans la presse, DOI: https://doi.org/10.1016/j.jbusres.2019.02.025.

Dampérat, M., Jeannot, F., Jongmans, E., et Jolibert, A. (2019), Modeling a cocreative process: The contributions of design and management, Recherche et Applications en Marketing, 34(3): 111-137.

Daunt, K. et Harris, L. (2017), Customer showrooming: Value co-destruction, Journal of Retailing and Consumer Services, 38(septembre): 166-176.

Delpechitre, D., Beeler-Connelly, L., et Chaker, N. (2018), Customer value co-creation behavior: A dyadic exploration of the influence of salesperson emotional intelligence on customer participation and citizenship behavior, Journal of Business Research, 92(novembre): 9-24.

Dong, B., Evans, K., et Zou, S. (2008), The effects of customer participation in co-created service recovery, Journal of the Academy of Marketing Science, 36(1): 123-137.

Dong, B. et Sivakumar, K. (2017), Customer participation in services: Domain, scope, and boundaries, Journal of the Academy of Marketing Science, 45(6): 944-965.

Drucker, P. (2011), Technology, Management, and Society, Harvard Business Review Press.

Echeverri, P. et Skålén, P. (2011), Co-creation and co-destruction: A practice-theory based study of interactive value formation, Marketing Theory, 11(3): 351-373.

Edvardsson, B., Tronvol, B., et Gruber, T. (2011), Expanding understanding of service exchange and value co-creation: A social construction approach, Journal of the Academy of Marketing Science, 39(2): 327-339.

Fisher, R. (1971), The Design of Experiments, MacMillan.

Fournier, A. (2019). My Starbucks idea: An open innovation case study (mars 20). Accédé (7 juin 2020): https://www.braineet.com/blog/my-starbucks-idea-case-study/.

Füller, J. (2010), Refining virtual co-creation from a consumer perspective, California Management Review, 52(2): 98.

Gebauer, J., Füller, J., et Pezzei, R. (2013), The dark and the bright side of co-creation: Triggers of member behavior in online innovation communities, Journal of Business Research, 66(9): 1516-1527.

Go Jefferies, J., Bishop, S., et Hibbert, S. (2019), Service innovation through resource integration: An empirical examination of co-created value using telehealth services, Public Policy and Administration, Dans la presse, DOI: https://doi.org/10.1177/0952076718822715.

Gooch, D. et Watts, L. (2015), The impact of social presence on feelings of closeness in personal relationships, Interacting with Computers, 27(6): 661-674.

Goudey, A. et Bonnin, G. (2016), Must smart objects look human? Study of the impact of anthropomorphism on the acceptance of companion robots, Recherche et Applications en Marketing, 31(2): 2-20.

Grace, D. et Iacono, J. (2015), Value creation: An internal customer's perspective, Journal of Services Marketing, 29(6/7): 560-570.

Granovetter, M. (1983), The strength of weak ties: A network theory revisited, Sociological Theory, 1: 201-233.

Grissemann, U. et Stokburger-Sauer, N. (2012), Customer co-creation of travel services: The role of company support and customer satisfaction with the co-creation performance, Tourism Management, 33(6): 1483-1492.

Grönroos, C. et Voima, P. (2013), Critical service logic: Making sense of value creation and co-creation, Journal of the Academy of Marketing Science, 41(2): 133-150.

Gummesson, E. (2011), Total Relationship Marketing, BH Publishing.

Gummesson, E. et Mele, C. (2010), Marketing as value co-creation through network interaction and resource integration, Journal of Business Market Management, 4(4): 181-198. 
Gunawardena, C. et Zittle, F. (1997), Social presence as a predictor of satisfaction within a computer-mediated conferencing environment, American Journal of Distance Education, 11(3): 8-26.

Gyrd-Jones, R. et Kornum, N. (2013), Managing the co-created brand: Value and cultural complementarity in online and offline multi-stakeholder ecosystems, Journal of Business Research, 66(9): 1484-1493.

Hair, J., Black, W., Babin, B., et Anderson, R. (2018), Multivariate Data Analysis, Pearson.

Hambrick, D. (1984), Taxonomic approaches to studying strategy: Some conceptual and methodological issues, Journal of Management, 10(1): 27-41.

He, W. et Yan, G. (2015), Mining blogs and forums to understand the use of social media in customer co-creation, Computer Journal, 58(9): 1909-1920.

Heidenreich, S., Wittowski, K., Handrich, M., et Falk, T. (2014), The dark side of customer co-creation: Exploring the consequences of failed co-created services, Journal of the Academy of Marketing Science, 43(3): 279-296.

Heitz, C., Blume, M., Scherrer, C., Stöckle, R. et Bachmann, T. (2019), Designing value co-creation for a free-floating ebike sharing system, INFORMS-CSS 2019: Smart Service Systems, Operations Management, and Analytics: 113-125.

Helm, C. et Jones, R. (2010), Extending the value chain - A conceptual framework for managing the governance of cocreated brand equity, Journal of Brand Management, 17(8): 579-589.

Hill, J. (2016), The impact of emojis and emoticons on online consumer reviews, perceived company response quality, brand relationship, and purchase intent, Accédé (7 juin 2020): https://scholarcommons.usf.edu/etd/6513/.

Hollebeek, L. (2019), Developing business customer engagement through social media engagement-platforms: An integrative S-D logic/RBV-informed model, Industrial Marketing Management, 81(août): 89-98.

Hollebeek, L., Clark, M., Andreassen, T., Sigurdsson, V., et Smith, D. (2020a), Virtual reality through the customer journey, Journal of Retailing \& Consumer Services, Dans la presse, DOI: https://doi.org/10.1016/j.jretconser.2020.102056.

Hollebeek, L., Sprott, D., et Brady, M. (2020b), Rise of the machines? Customer engagement in automated service interactions, Document de travail.

Hollebeek, L., Srivastava, R., et Chen, T. (2019), S-D logic-informed customer engagement: Integrative framework, revised fundamental propositions, and application to CRM, Journal of the Academy of Marketing Science, 47(1): 161-185.

Hopkins, J. (2020), The concept of affordances in digital media, Handbuch Soziale Praktiken und Digitale Alltagswelten, 47-54.

Huang, M.H. et Rust, R. (2018), Artificial intelligence in service, Journal of Service Research, 21(2): 155-172.

Huang, M.H. et Rust, R. (2020), Engaged to a robot? The role of AI in service, Journal of Service Research, Dans la presse, DOI: https://doi.org/10.1177/1094670520902266.

Huber, T., Kude, T. et Dibbern, J. (2017). Governance practices in platform ecosystems: Navigating tensions between cocreated value and governance costs. Information Systems Research, 28(3): 451.

Hult, G.T., Mena, J., Ferrell, O.C., et Ferrell, L. (2011), Stakeholder marketing: A definition and conceptual framework, AMS Review, 1(1): 44-65.

Humphreys, A. et Grayson, K. (2008), The intersecting roles of consumer and producer: A critical perspective on coproduction, co-creation and presumption, Sociology Compass, 2(3): 963-980.

Kannan, P. et Li, H. (2017), Digital marketing: A framework, review and research agenda, International Journal of Research in Marketing, 34: 22-45.

Keeling, D., De Ruyter, K., Mousavi, S., et Laing, A. (2019), Technology push without a patient pull: Examining digital unengagement (DU) with online health services, European Journal of Marketing, 53(9): 1701-1732.

Kim, S., Schmitt, B., et Thalmann, N. (2019), Eliza in the uncanny valley: Anthropomorphizing consumer robots increases their perceived warmth but decreases liking, Marketing Letters, 30, 1-12.

Kitano, N. (2006), Rinri: An incitement towards the existence of robots in Japanese society, Ethics in Robotics, 6(12), Accédé: http://citeseerx.ist.psu.edu/viewdoc/download?doi=10.1.1.115.9957\&rep=rep1\&type=pdf\#page=80.

Kohler, T., Fueller, J., Stieger, D., et Matzler, K. (2011), Avatar-based innovation: Consequences of the virtual co-creation experience, Computers in Human Behavior, 27(1): 160-168.

Kowalski, R., Limber, S., et Agatston, P. (2012), Cyberbullying: Bullying in the Digital Age, Blackwell.

Kristensson, P., Matthing, J., et Johansson, N. (2008), Key strategies for the successful involvement of customers in the cocreation of new technology-based services, International Journal of Service Industry Management, 19(4): 474-491.

Kull, A. et Heath, T. (2016), You decide, we donate: Strengthening consumer-brand relationships through digitally cocreated social responsibility, International Journal of Research in Marketing, 33: 78-92.

Kumar, V., Dixit, A., Javalgi, R. et Dass, M. (2016). Research framework, strategies, and applications of intelligent agent technologies (IATs) in marketing. Journal of the Academy of Marketing Science, 44(1): 24-45.

Larivière, B., Bowen, D., Andreassen, T., Kunz, W., Sirianni, N., Voss, C., Wünderlich, N., et De Keyser, A. (2017), Service encounter 2.0: An investigation into the roles of technology, employees, and customers, Journal of Business Research, 79(octobre): 238-246.

Leclercq, T., Hammedi, W., et Poncin, I. (2016), Ten years of value cocreation: An integrative review, Recherche et Applications en Marketing, 31(3): 26-60. 
Lee, S., Olson, D. et Trimi, S. (2012), Co-innovation: Convergonomics, collaboration, and co-creation for organizational values, Management Decision, 50(5): 817-831.

Lee, S. et Sathikh, P. (2013), A framework for effective human-to-machine communication for artificial intelligence systems, International Conference on Engineering Design: Human Behavior in Design.

Leicht, T., Chtourou, A., et Youssef, K. (2018), Consumer innovativeness and intentioned autonomous car adoption, Journal of High Technology Management Research, 29(1): 1-11.

Lenka, S., Varida, V., et Wincent, J. (2017), Digitalization capabilities as enablers of value co-creation in servitizing firms, Psychology \& Marketing, 34(1): 92-100.

Li, L., Huang, Q., et Jian, Z. (2018), Human-computer interaction and value co-creation in electronic service, Industrial Management \& Data Systems, 118(1): 218-235.

Lombard, M. et Ditton, T. (1997), At the heart of it all: The concept of presence, Journal of Computer-Mediated Communication, 3(2): Accédé (1 juin 2020): https://academic.oup.com/jcmc/article/3/2/JCMC321/4080403.

Lovelock, M. (2017), Catching a catfish: Constructing the 'good' social media user in reality television, Television \& New Media, 18(3), 203-217.

Luangrath, A., Peck, J., et Barger, V. (2017), Textual paralanguage and its implications for marketing communications, Journal of Consumer Psychology, 27(1): 98-107.

Lusch, R., Vargo, S., et O’Brien, M. (2007), Competing through service: Insights from service-dominant logic, Journal of Retailing, 83(1): 5-18.

Lv, Z., Jin, Y., et Huang, J. (2018), How do sellers use live chat to influence consumer purchase decision in China? Electronic Commerce Research and Applications, 28(mars/avril): 102-113.

MacInnis, D. (2011), A framework for conceptual contributions in marketing, Journal of Marketing, 75(juillet): 136-154.

Mahr, D., Lievens, A. et Blazevic, V. (2014), The value of customer cocreated knowledge during the innovation process, Journal of Product Innovation Management, 31(3): 599-615.

Marin, L., Issartel, J. et Chaminade, T. (2009), Interpersonal motor coordination: From human-human to human-robot interactions, Interaction Studies, 10(3): 479-504.

Marr, B. (2018). Artificial intelligence in business. Accédé (3 mai 2020): https://www.bernardmarr.com/default.asp?contentID=1314.

Maslow, A. (1954), Motivation and Personality, Harper \& Row.

McColl-Kennedy, J., Vargo, S., Dagger, T., Sweeney, J., et Van Kasteren, Y. (2012), Health care customer value cocreation practice styles, Journal of Service Research, 15: 370-389.

Mende, M., Scott, M., Van Doorn, J., Grewal, D., et Shanks, I. (2019), Service robots rising: How humanoid robots influence service experiences and elicit compensatory consumer responses, Journal of Marketing Research, 56(4): 535-556.

Merz, M., Zarantonello, L., et Grappi, S. (2018), How valuable are your customers in the brand value co-creation process? The development of a customer co-creation value (CCCV) scale, Journal of Business Research, 82: 79.

Mossberg, L., Hanefors, M. et Hansen, A. (2014), Guide performance: Co-created experiences for tourist immersion, In: Prebensen, N., Chen, J. et Uysal, M. (Eds.), Creating Experience Value in Tourism: 234-247.

Nakanishi, H., Kato, K., and Ishiguro, H. (2011), Zoom cameras and movable displays enhance social telepresence, CHI '11/SIGCHI Conference: Human Factors in Computing Systems: 63-72.

Ng, I., Maull, R., et Smith, L. (2011), Embedding the new discipline of service science, In: Demirkan, H., Spohrer, J. et Krishna, V. (Eds.), Service Science, Springer, 13-36.

$\mathrm{Ng}$, I. et Wakenshaw, S. (2017), The Internet-of-Things: Review and research directions, International Journal of Research in Marketing, 34(1): 3-21.

Nicod, L. et Llosa, S. (2018), How should customers be trained in their role as coproducers? The influence of training and its characteristics on the benefits of coproduction, Recherche et Applications en Marketing, 33(4): 46-73.

Normann, R. et Ramírez, R. (1994), Designing Interactive Strategy, Wiley.

Novani, S. et Kijima, K. (2012), Value co-creation by customer-to-customer communication: Social media and face-to-face for case of airline service selection, Journal of Service Science and Management, 5(1): 1-5.

Oertzen, A., Odekerken-Schröder, G., Brax, S., et Mager, B. (2018), Co-creating services: Conceptual clarification, forms and outcomes, Journal of Service Management, 29(4): 641-679.

Osei-Frimpong, K. et McLean, G. (2018), Examining online social brand engagement: A social presence theory perspective, Technological Forecasting and Social Change, 128(mars): 10-21.

Pirrone, D., Andolina, S., Santangelo, A., Gentile, A., et Takizawa, M. (2012), Platforms for human-human interaction in large social events, IEEE Xplore, Accédé: https://ieeexplore.ieee.org/abstract/document/6363113.

Plé, L. et Chumpitaz Cáceres, R. (2010), Not always co-creation: Introducing interactional co-destruction of value in servicedominant logic, Journal of Services Marketing, 24(6): 430-437.

Pradeep, A., Appel, A., et Sthanunathan, S. (2019), AI for Marketing and Product Innovation, Wiley.

Prahalad, C. et Krishnan, M. (2008), The New Age of Innovation, McGraw-Hill.

Prahalad, C. et Ramaswamy, V. (2000), Co-opting customer competence, Harvard Business Review, 78(1): 79-90.

Prahalad, C. et Ramaswamy, V. (2004), Co-creation experiences: The next practice in value creation, Journal of Interactive Marketing, 18(3): 5-14. 
Raïes, K. et Gavard-Perret, M.L. (2011), Brand loyalty intention among members of a virtual brand community: The dual role of commitment, Recherche et Applications en Marketing, 26(3): 23-41.

Ramaswamy, V. et Ozcan, K. (2016), Brand value co-creation in a digitalized world: An integrative framework and research implications, International Journal of Research in Marketing, 33(1): 93-106.

Ramaswamy, V. et Ozcan, K. (2018), What is co-creation? An interactional creation framework and its implications for value creation, Journal of Business Research, 84: 196-205.

Ranjan, K. et Read, S. (2016), Value co-creation: Concept and measurement, Journal of the Academy of Marketing Science, 44(3): 290-315.

Rice, R. (1993), Media appropriateness: Using social presence theory to compare traditional and new organizational media, Human Communication Research, 19(4): 451-484.

Saha, V., Hollebeek, L., Venkatesh, M., et Goyal, P. (2020), Cocreation, Document de travail.

Schau, H., Muniz, A., et Arnould, E. (2009), How brand community practices create value, Journal of Marketing, 73(5): 3051.

Short, J., Williams, E., et Christie, B. (1976), The Social Psychology of Telecommunications. London: John Wiley.

Song, J.H. et Hollenbeck, C. (2015), The value of social presence in mobile communications, Service Industries Journal, 35(11-12), 611-632.

Song, J.H., Hollenbeck, C., et Zinkhan, G. (2008), The value of human warmth: Social presence cues and computer-mediated communications, In: Lee, A. and Soman, D. (Eds.), North American Advances in Consumer Research, 35, 793-794.

Schweitzer, F., Belk, R., Jordan, W., et Ortner, M. (2019), Servant, friend or master? The relationships users build with voice-controlled smart devices, Journal of Marketing Management, 35(7/8): 693-715.

Smedlund, A. (2012), Value cocreation in service platform business models. Service Science, 4(1): 78-88.

Smedlund, A. et Faghankhani, H. (2015), Platform orchestration for efficiency, development, and innovation, Hawaii International Conference on Systems Science, Accédé (7 mai 2020): https://ieeexplore.ieee.org/xpl/conhome/7068092/proceeding.

Smith, A. (2013), The value co-destruction process: A customer resource perspective, European Journal of Marketing, 47(11-12): 1889-1909.

Täuscher, K. et Laudien, S. (2017), Understanding platform business models: A mixed methods study of marketplaces, European Management Journal, 36(3): 319-329.

Tixier, M., Lewkowicz, M., Maroccia, M., Atifi, H., Bénel, A., Gaglio, G., et Gauducheau, N. (2010), Practices analysis and digital platform design: An interdisciplinary study of social support, COOP2010, 309-329.

Troisi, O., D’Arco, M., Loia, F., et Maione, G. (2018), Big data management: The case of Mulino Bianco's engagement platform for value co-creation. International Journal of Engineering Business Management, DOI: https://doi.org/10.1177/1847979018767776.

$\mathrm{Tu}, \mathrm{C}$. (2000), On-line learning migration: From social learning theory to social presence theory in a CMC environment, Journal of Network and Computer Applications, 23(1): 27-37.

Tu, J. et Zhang, M. (2013), Research on the effect of co-creation customer experience on customer co-created value in nontrading virtual community, IEEE International Conference: Service Operations and Logistics, Accédé (24 juin 2020): https://ieeexplore.iee. org/abstract/document/6611395.

Uhrich, S. (2014), Exploring customer-to-customer value cocreation platforms and practices in team sports, European Sports Management Quarterly, 14(1): 25-49.

Van Doorn, J., Mende, M., Noble, S., Hulland, J., et al. (2017), Domo arigato Mr. Roboto: Emergence of automated social presence in organizational frontlines and customers' service experiences, Journal of Service Research, 20(1): 43.

Vanhouette, E. (2016). The gates of hell: History and definition of digital | humanities | computing. In: Terras, M., Nyhan, J. et Vanhouette, E. (Eds.), Defining Digital Humanities, 119-156, Taylor \& Francis.

Vargo, S. et Lusch, R. (2004), Evolving to a new dominant logic for marketing, Journal of Marketing, 68(1): 1-17.

Vargo, S. et Lusch, R. (2008), Service-dominant logic: Continuing the evolution, Journal of the Academy of Marketing Science, 36(1): 1-10.

Vargo, S. et Lusch, R. (2010), From repeat patronage to value co-creation in service ecosystems: A transcending conceptualization of relationship. Journal of Business Market Management, 4: 169-179.

Vargo, S. et Lusch, R. (2016), Institutions and axioms: An extension and update of service-dominant logic, Journal of the Academy of Marketing Science, 44(1): 5-23.

Vega-Vazquez, M., Revilla-Camacho, A., et Cossio-Silva, J. (2013), The value co-creation process as a determinant of customer satisfaction, Management Decision, 51(10): 1945-1953.

Verma, R., Gustafsson, A., et Witell, L. (2012), Customer co-creation in service innovation: A matter of communication? Journal of Service Management, 23(3): 311-327.

Voss, K., Spangenberg, E., et Grohmann, B. (2003), Exploring the hedonic and utilitarian dimensions of consumer attitude, Journal of Marketing Research, 40(3): 310-320.

Weyerer, J. et Langerer, P. (2019), Garbage in, garbage out: The vicious cycle of AI-based discrimination in the public sector, Annual International Conference: Digital Government Research, 509-511. 
Wiener, M. et Mehrabian, A. (1968), Language within Language: Immediacy, a Channel in Verbal Communication, Ardent Media.

Willcocks, L., Lacity, M., et Craig, A. (2015), Robotic process automation at Xchanging, The Outsourcing Unit Working Paper Series, Accédé (1 mai 2020): http://eprints.lse.ac.uk/64518/1/OUWRPS_15_03_published.pdf.

Wirtz, J., Patterson, P., Kunz, W., Gruber, T., Lu, V., Paluch, S., et Martins, A. (2018), Brave new world: Service robots in the frontline, Journal of Service Management, 29(5): 907-931.

Witell, L., Kristensson, P., Gustafsson, A. et Lofgren, M. (2011), Idea generation: Customer co-creation versus traditional market research techniques, Journal of Service Management, 22(2): 140-159.

Woodruff, R. (1997), Customer value: The next source for competitive advantage, Journal of the Academy of Marketing Science, 25(2): 139-153.

Wu, Y., Wu, C., Li, B., Zhang, L., Li, Z., et Lau, F. (2014), Scaling social media applications into geo-distributed clouds, IEEE/ACM Transactions on Networking, Accédé (22 juin 2020): https://core.ac.uk/download/pdf/38052757.pdf.

Wünderlich, N., Wangenheim, F., et Bitner, M.J. (2013), High tech and high touch: A framework for understanding user attitudes and behaviors related to smart interactive services. Journal of Service Research, 16(1): 3-20.

Yadav, M. (2010), The decline of conceptual articles and implications for knowledge development, Journal of Marketing, 74(1): 1-19.

Zeithaml, V. (1988), Consumer perceptions of price, quality, and value: A means-end model and synthesis of evidence, Journal of Marketing, 52(juillet): 2-22.

Zhang, T., Lu, C., Torres, E., et Chen, P. (2018), Engaging customers in value co-creation or co-destruction online, Journal of Services Marketing, 32(1): 57-69. 